\title{
Rapid Explorative Direct Inverse Kinematics Learning of Relevant Locations for Active Vision
}

\author{
Kristoffer Öfjäll and Felsberg Michael
}

\section{Linköping University Post Print}

N.B.: When citing this work, cite the original article.

(C2013 IEEE. Personal use of this material is permitted. However, permission to reprint/republish this material for advertising or promotional purposes or for creating new collective works for resale or redistribution to servers or lists, or to reuse any copyrighted component of this work in other works must be obtained from the IEEE.

Kristoffer Öfjäll and Felsberg Michael, Rapid Explorative Direct Inverse Kinematics Learning of Relevant Locations for Active Vision, 2013, IEEE Workshop on Robot Vision(WORV) 201 . 


\title{
Rapid Explorative Direct Inverse Kinematics Learning of Relevant Locations for Active Vision
}

\author{
Kristoffer Öfjäll, Michael Felsberg \\ Linköping University \\ Linköping, Sweden \\ \{kristoffer.ofjall, michael.felsberg\}@liu.se
}

\begin{abstract}
An online method for rapidly learning the inverse kinematics of a redundant robotic arm is presented addressing the special requirements of active vision for visual inspection tasks. The system is initialized with a model covering a small area around the starting position, which is then incrementally extended by exploration. The number of motions during this process is minimized by only exploring configurations required for successful completion of the task at hand. The explored area is automatically extended online and on demand.

To achieve this, state of the art methods for learning and numerical optimization are combined in a tight implementation where parts of the learned model, the Jacobians, are used during optimization, resulting in significant synergy effects. In a series of standard experiments, we show that the integrated method performs better than using both methods sequentially.
\end{abstract}

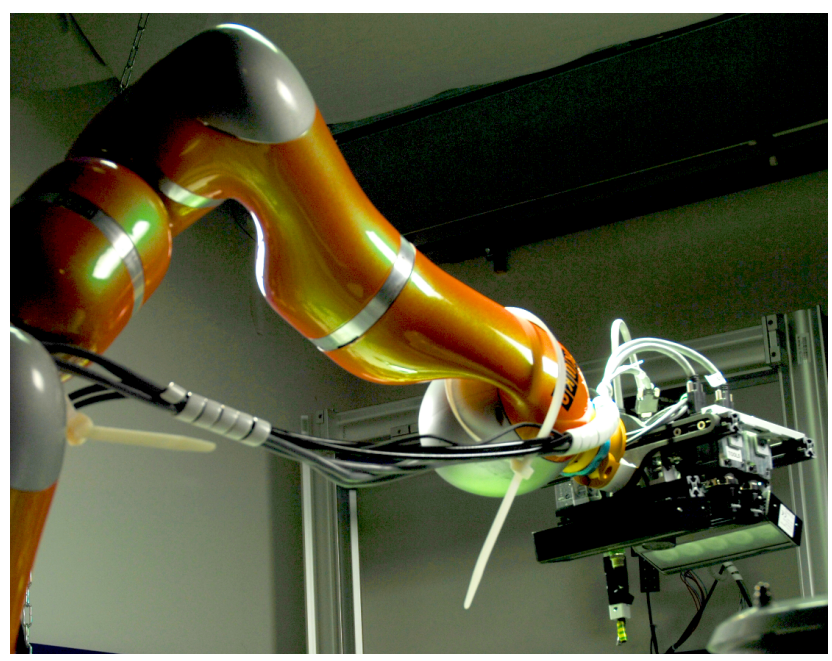

Figure 1. Robotic arm with mounted cameras for active visual inspection.

\section{Introduction}

A popular approach to active vision is to mount cameras at the end effector of a robotic arm. In such systems, the desired camera pose is expressed as position and orientation in a Cartesian coordinate system. Finding joint configurations resulting in a requested pose of the end effector (camera) is known as inverse kinematics.

Several methods for inverse kinematics, based on physical modeling [16] or learning [5], have been proposed in the literature, but these ignore the special requirements of active vision applications. In active vision, the system requests images from certain camera poses and the requested poses have a tendency to reoccur close to previously visited poses. One example of such an application is active visual inspection, Fig. 1, where the system usually views the inspected objects from roughly the same positions, but occasionally may need additional views to successfully inspect a particular object. Monitoring plants is a good example requiring explorative learning, since plants might leave the assumed shape or size range, thus forcing the robot to move outside the expected area.

Using learning approaches for robot control has advantages such as the ability to adapt to changes in the robot which could be induced by e.g. wear or maintenance. General learning controllers for robotic arms aim to build an accurate model of the arm either in the whole reachable space [2], or along the trajectories where the arm is supposed to move [13]. Such controllers, directly applied in an active vision context, thus waste many iterations of arm movements to learn precise models of the robotic arm in areas where high accuracy is not needed.

In active vision applications, the images acquired by the camera can be used to iteratively approach a desired pose by reducing the pose error in a closed visual servoing loop. Obviously emphasis is on accurately reaching the desired pose with as few iterations of moving the robotic arm as possible.

The main contribution of this paper is the combination 
of a machine learning method, such as LWPR [18], with a numerical optimization method, such as LM [8]. Machine learning provides a possibility to directly return to previously visited areas while optimization enables efficient explorative learning and automatic training data generation. Initial solutions for the optimization is provided by the learning algorithm. We demonstrate the benefits of such a combination by proposing a method for achieving high control accuracy to relevant poses with few iterations.

A direct combination of the two methods already performs well but as each of the two algorithms internally generates information that would be useful for the other, performance is expected to improve in a tight integration of both methods. In this paper, these two approaches are compared. The paper is structured as follows: Section 2 provides an overview over existing methods, Section 3 explains our proposed approach, Section 4 describes the evaluation method with the results reported in Section 5, and Section 6 concludes the paper.

\section{Previous Work}

To the best of our knowledge, there has not been any proposed solutions to the inverse kinematics learning for active vision problem in the literature. Proposed solutions to related inverse kinematics problems are presented in this section. The deterministic way of solving inverse kinematic problems uses handcrafted geometric models of the robots. The accuracy depends on the complexity of the model and any changes of the robot behavior due to wear are disregarded.

Control models based on learning systems have the possibility to adapt to individual differences between robots of the same manufacturer and type as well as being able to learn different robot setups. By retraining the system, also changes in the robot can be handled. Different variations of neural networks have been popular approaches to this problem [11]. The results have been improved by using modular neural networks $[9,10]$, where several different neural networks are trained and the output from the locally most suitable network is used.

The neural network approaches mostly use offline training and require training points in the order of millions. Depending on the physical layout of the robotic arm, the mapping can be decomposed into one mapping from orientation of the end effector to a subset of the joints and another mapping from position to the remaining joints [1]. Changes in the robot still require offline retraining.

\subsection{Online Learning}

With online learning methods, the robot model can adapt to changes while the robot is in use. This has shown to be useful, especially in cases where the robotic arm tends to change properties within short time frames such as in [6].
Online learning requires the system to be able to measure the resulting pose when a given configuration has been commanded. In vision applications the pose may be deduced from the captured images. This is fundamental in visual servoing and in combination with online kinematics learning this enables continuous improvement of the kinematics model. If a (possibly low accuracy) geometric model is available, it may be used to initialize the learnt model as proposed in [4].

Pure learning methods encounter problems when not previously visited poses are requested. Motor babbling is a popular approach where random motions around the current configuration are carried out. In some cases this would provide information on how to proceed towards the desired pose, but as described in [14], falling down might not tell us much about the forces needed in walking. That is, motor babbling may generate training data that does not affect the predictions of the learnt model.

\subsection{Active Learning}

Active learning generally means that the learning algorithm can affect the generation or selection of training data. The term is not clearly defined but [15] provides a survey. In our case, the system has the possibility to actively control the robot. This can be used to generate training data that maximizes information gain given the current state of the model.

Furthermore, these strategies can be combined with motor babbling as in [12] or [2] where goals are used to direct the babbling and exploration of the available motion space. To some extent, this addresses the problem of generating useful training data present in pure motor babbling methods. Goals are automatically generated to explore the whole space. [3] presents a more general and theoretical framework for this type of active learning, on a statistical foundation.

When the space of possible movements is large, exploring the whole space can be very time consuming and depending on the application, only a small subset of these movements may be used. The Shifting Setpoint Algorithm [13] is a method where models are built along tubes in the motion space between desired points. As the name suggests, a setpoint is shifted towards the desired point and motor babbling is carried out around it. When the model is good enough locally (as determined by the algorithm), the setpoint is shifted again. This solves a similar problem but robot movement iterations and time are unnecessarily spent generating accurate models in between desired poses.

\subsection{Numerical Optimization Methods}

Viewing the inverse kinematics problem as minimizing a distance between the current and desired poses enables the use of algorithms from the large field of optimization [17]. 
Numerical optimization methods geared towards reaching a minimum using as few iterations as possible will avoid unnecessary movements of the robot.

If the kinematics of the robotic arm is not known, a numerical method has to be used. This implies iterative or grid based methods, where the former is expected to require fewer movements of the arm. One popular numerical algorithm is the Levenberg-Marquardt method [8]. For fast convergence, the algorithm requires a good initial solution and the derivative of the distance function at the iteration points. One possibility is to use the current pose of the robot as initial solution and estimating the derivatives using finite differences. As will be shown, there are better ways of choosing the initial solution as well as obtaining derivatives.

In Gauss-Newton optimization, the update $\mathbf{q}_{\Delta}$ in each step is obtained by solving a linearized problem

$$
\mathbf{J}^{T} \mathbf{J} \mathbf{q}_{\Delta}=-\mathbf{J}^{T} \mathbf{e}
$$

where $\mathbf{J}$ is the Jacobian at the current point and $\mathbf{e}$ is the current residual vector. In LM, the joint space update $\mathbf{q}_{\Delta}$ in each step is obtained by solving

$$
\left(\mathbf{J}^{T} \mathbf{J}+\lambda \operatorname{diag}\left(\mathbf{J}^{T} \mathbf{J}\right)\right) \mathbf{q}_{\Delta}=\mathbf{J}^{T}\left(\mathbf{x}_{d}-\mathbf{x}(\mathbf{q})\right)
$$

where $\mathbf{J}$ is the Jacobian at the current configuration $\mathbf{q}, \mathbf{x}(\mathbf{q})$ is the pose of the current configuration and $\mathbf{x}_{d}$ is the desired pose. The method is a weighted (by $\lambda$ ) combination of Gauss-Newton and weighted gradient descent.

\subsection{Forward and Inverse Kinematics}

For a serial robotic arm such as in Fig. 1, the forward kinematics is injective and a rigid body model can be deduced from the physical layout of the arm. Most methods, learning or geometric, model the forward kinematics and use some algorithm to solve the inverse kinematic problem.

For these serial manipulators, the mapping from pose space to joint space, the inverse kinematics, is not injective as different joint configurations may result in the same pose of the end effector. As stated in [5], it is not possible to learn the full inverse mapping using methods which generate averages of different configurations resulting in the same pose. However, if the learning system is only exposed to one solution, this issue do not arise.

\subsection{Locally Weighted Projection Regression}

Locally Weighted Projection Regression [18], LWPR, has successfully been applied to learning both forward kinematics [13], and direct inverse kinematics with spatial localization [5]. The general idea is to use the output from several local linear models weighted together to form the output.
The output $y_{d k}$ for each local model $k$ for dimension $d$ consists of $r_{k}$ linear regressors

$$
y_{d k}=\beta_{d k}^{0}+\sum_{i=1}^{r_{k}} \beta_{d k i} \mathbf{u}_{d k i}^{T}\left(\mathbf{x}_{d k i}-\mathbf{x}_{d k}^{0}\right)
$$

along different directions $\mathbf{u}_{d k i}$ in the input space. Each projection direction and corresponding regression parameter $\beta_{d k i}$ and bias $\beta_{d k}^{0}$ are adjusted online to fit the training examples. Variations in the input explained by each regression $i$ is removed from the input $\mathbf{x}$ generating the input to the next regressor $\mathbf{x}_{d k(i+1)}$.

The total prediction $\hat{y}_{d}$ in one output dimension $d$

$$
\hat{y}_{d}=\frac{\sum_{k=1}^{K} w_{d k} y_{d k}}{\sum_{k=1}^{K} w_{d k}}
$$

depends on the distance from the center $\mathbf{c}_{d k}$ of each of the local models. Normally a Gaussian kernel is used, generating the weights

$$
w_{d k}=\exp \left(-\frac{1}{2}\left(\mathbf{x}-\mathbf{c}_{d k}\right)^{T} \mathbf{D}_{d k}\left(\mathbf{x}-\mathbf{c}_{d k}\right)\right)
$$

where the metric $\mathbf{D}_{d k}$ is updated while the model centers $\mathbf{c}_{d k}$ remain constant. An advantage of LWPR which will be used later, is the possibility to derive analytic Jacobians of the learnt model [7].

\section{Proposed Method}

By combining an online learning approach with a numerical optimization method, the desired pose is reached quicker on average. The current learnt model can be used to provide an initial solution as well as estimated Jacobians as required by numerical optimization methods. In return, the iterations of the numerical method serves as excellent training data along the lines of active learning.

In this paper, a combination of LWPR and LevenbergMarquardt, LM, is evaluated. The proposed integration of both methods is compared with a naïve combination of the two algorithms. In the baseline implementation, only outputs from the two algorithms are used. For the integrated method, internal data is shared.

\subsection{Initial Solution}

In the LWPR algorithm, a weight threshold $w_{\text {cut }}$ is used to determine which local models should be used in (4). This can also be used to determine where reasonable predictions can be expected. Given the weight of each local model (5), using the notation of (5), reasonable predictions can be expected within the set

$$
X_{p}=\bigcap_{d}\left(\bigcup_{k}\left\{\mathbf{x}:\left(\mathbf{x}-\mathbf{c}_{d k}\right)^{T} \mathbf{D}_{d k}\left(\mathbf{x}-\mathbf{c}_{d k}\right) \leq-\ln w_{c u t}\right\}\right)
$$


as at least one local model $k$ in each output dimension $d$ should provide a useful prediction. Given a desired pose $\mathbf{x}_{d}$ it is thus possible to find an initial pose $\mathbf{x}_{t} \in X_{p}$ such that no other pose $\mathbf{x} \in X_{p}$ is closer to the desired pose.

An approximation of the optimal initial pose $\mathbf{x}_{t}$ still within $X_{p}$ can easily be found by starting in the desired pose $\mathbf{x}_{d}$ and using gradient ascent on

$$
\min _{d}\left(\max _{k} \exp \left(-\left(\mathbf{x}-\mathbf{c}_{d k}\right)^{T} \mathbf{D}_{d k}\left(\mathbf{x}-\mathbf{c}_{d k}\right)\right)\right)
$$

to improve the dimension with the worst response of the best fitting model. The ascent is continued until the value of the expression reaches $w_{c u t}$ where the learnt inverse kinematics model is expected to be accurate enough. The model can then be used to move the arm directly close to this pose. As the initial pose may be on the border of $X_{p}$ (the set of poses where inverse kinematic predictions can be made) the accuracy of the inverse kinematics model can not be expected to be perfect.

If the robotic arm has previously moved close to the desired pose, the initial pose will be very close to the desired pose. In areas where the inverse kinematics model is accurate, the desired pose will be reached directly.

\subsection{Pose Optimization}

The desired pose can be reached by directly minimizing the pose error $e\left(\mathbf{x}_{d}-\mathbf{x}(\mathbf{q})\right)$ with current pose $\left.\mathbf{x}(\mathbf{q})\right)$ and desired pose $\mathbf{x}_{d}$ using LM, (2). Visited poses are available as training data for LWPR. As the robot kinematics is not known, the Jacobian has to be estimated or otherwise obtained. Using LM blindly, it is estimated using finite differences, but in the proposed integrated method, it is obtained from the LWPR model without moving the robot.

Using the regularization parameter $\lambda$ of LM, the step length can be limited as done in trust region methods. This is useful to prevent the robotic arm from maneuvering outside safe areas. As a side effect, if the desired pose cannot be reached, the optimization will result in a pose that is as close to the desired pose as the robot can get, where close is defined by the used pose distance measure.

\section{Evaluation}

As common in literature $[4,10,7,17]$, the presented methods are evaluated on a simulated, planar robotic arm. The arm has three joints and the desired pose is the two dimensional position of the tip of the last link. The evaluated method controls the arm by specifying the three joint angles and the simulation return the position of the final link with added Gaussian noise.

The direct difference between the current position and the desired position is used in the quadratic error function of the Levenberg-Marquardt algorithm implemented as
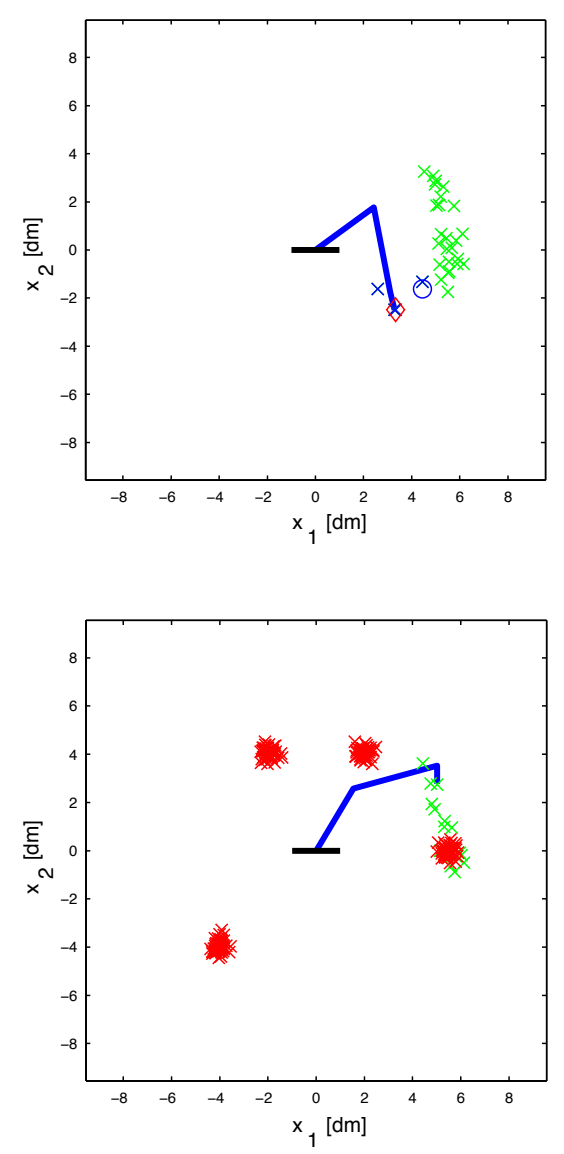

Figure 2. (Top) The simulated robotic arm reaching for the red diamond marker. The blue circle indicates the initial position, the blue crosses are the iterations and the green crosses are the initial training data. (Bottom) The evaluation points marked with red crosses.

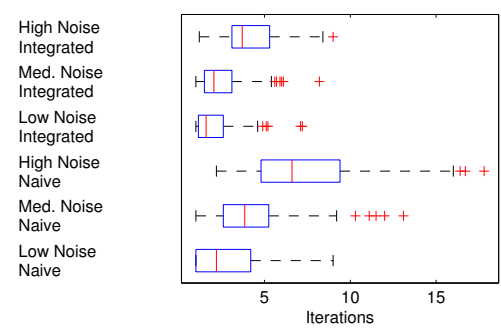

Figure 3. Quartiles of the number of iterations required to reach each of the last 100 evaluation points for each evaluation session.

lsqnonlin in Matlab. For LWPR, the implementation by Klanke, Vijayakumar and Schaal is used [18].

An initial training set with 15 points distributed in a small area of the possible movement area of the robot is generated. Evaluation points are generated in four clusters with 50 points in each cluster. The training and evaluation points are shown in Fig. $2 b$. 

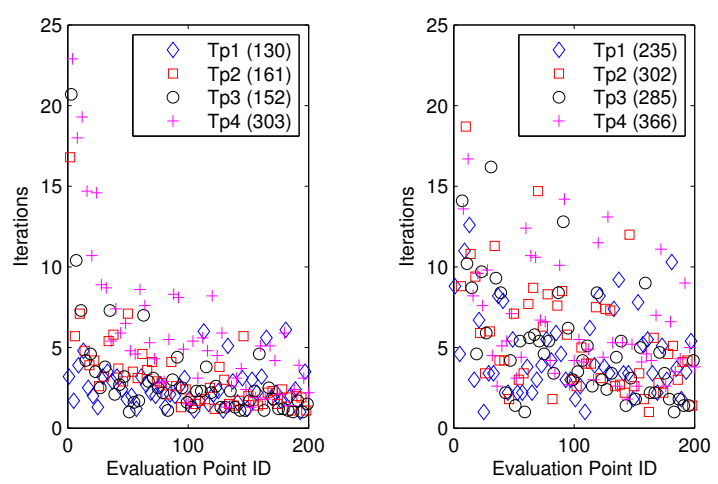

Figure 4. Mean number of iterations required to reach each evaluation point. (Left) Integrated method; (Right) Naïve combination.

For evaluation, each LWPR model is trained using the training points. Each method is then used to move to the first evaluation point in the first cluster and the number of iterations required to reach the point is counted. This is repeated for the first point in the second cluster and so on until all evaluation points are reached.

When the first evaluation point in a cluster far from the training data is to be reached, the system is expected to require some iterations to move from the closest previously visited area to the desired position. When the arm is supposed to return to the second point within the same cluster, the number of iterations required is expected to be lower. After visiting a few points within the cluster, the system is expected to be able to move directly to the desired pose.

This behavior is expected both for systems using numerically estimated Jacobians and for systems using Jacobians from the learnt model. For the Jacobians from the learnt model, the accuracy is expected to be low during the first runs but increasing as more points are visited within each cluster. For the numerically estimated Jacobians, the arm has to be moved for Jacobian estimation. Thus, if the system does not move the arm to the correct position in the first iteration, at least four additional movements of the arm are required. Three of which are required to estimate the Jacobian.

In contrast to the learning approaches, using a numerical optimization method alone, always starting at the current arm pose, the number of iterations required to reach each point could be expected to stay constant as no information regarding the behavior of the robot is kept.

\section{Results}

The evaluation results are shown in Fig. 4. The graphs show the number of iterations required to reach each test point for the naïve combination and the proposed integrated method respectively. We expect real measurements to be noisy so independent zero mean Gaussian noise with stan-
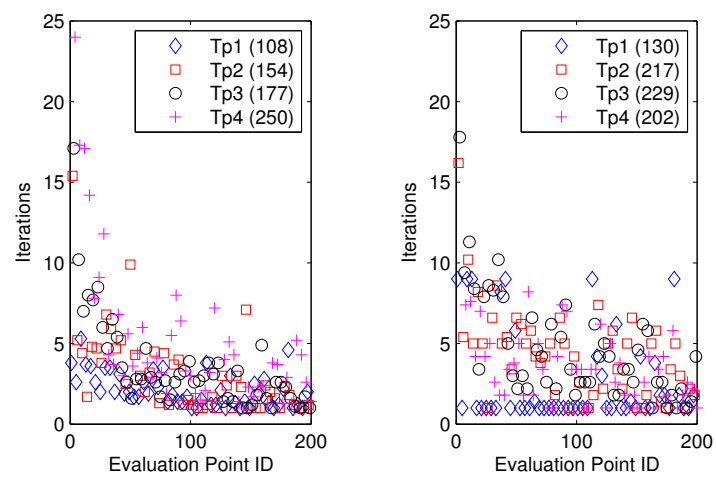

Figure 5. Mean number of iterations required to reach each evaluation point with reduced measurement noise. (Left) Integrated method; (Right) Naïve combination.
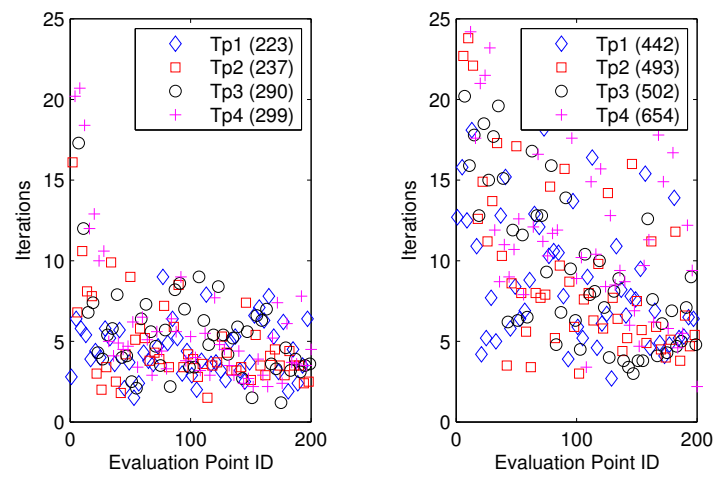

Figure 6. Mean number of iterations required to reach each evaluation point with increased measurement noise. (Left) Integrated method; (Right) Naïve combination.

dard deviation 0.03 was added to the position estimates. This corresponds to $0.2 \%$ of the diameter of the space reachable by the robot. The shown results are the average of ten runs. As expected, the number of iterations required to reach a point decays with increasing number of visited points in the same cluster.

If the Jacobians from the learnt model were perfect, the method using these should require about one fourth of the iterations required by the naïve method. Using Jacobians from the learnt model requires significantly fewer iterations than using numerically estimated Jacobians, but the theoretically possible reduction is not achieved. This is due to errors in the learnt model and to the implementation of the numerical solver not needing to estimate the Jacobian at every step.

Additionally, to assess the effect of the noise, simulations were run with reduced and increased amount of noise. Reducing the standard deviation to one sixth of the original noise, the results in Fig. 5 are obtained. In Fig. 6 the standard deviation of the noise is doubled.

In the case of reduced noise, both methods perform bet- 
ter. The integrated method is better than using numerically estimated Jacobians. Increasing the noise, the numerical method performs significantly worse. Here, the averaging introduced by the learning method is a great advantage. In Fig. 3 evaluation results for the last 100 points in each session are presented more compactly. For each noise level, t-tests on this data indicate sigificant improvements $\left(p_{H_{0}}<0.01\right)$ where $H_{0}$ : Equal mean error for naïve and integrated methods. One example of reaching a desired pose is shown in Fig. 2a.

\section{Conclusions}

We have presented a method for active inverse kinematics learning, geared towards active vision and visual inspection. While earlier methods have aimed for models with high precision in large parts of the reachable area of the robot, visual inspection only requires high precision on isolated small islands.

Since methods previously described in literature are not aimed towards the active visual inspection scenario, these methods would need modifications for a fair comparison. Numbers and results reported in the literature indicate that learning the whole pose space requires tens or hundreds of thousands of iterations. Methods limited to paths in pose space require tens of iterations around each of several points along these paths [13]. As we have shown, the proposed method can step through large distances in pose space in just a few iterations and concentrate the learning where a precise model is needed.

By close integration of state of the art methods, in particular by sharing the Jacobian, the average number of iterations required to reach a given pose is reduced significantly compared to blindly combining the same methods. We have shown this in a series of synthetic experiments, as usual in the literature on learning kinematics, but we have also used our learning method successfully on a real robotic arm.

\section{Acknowledgement}

This research has received funding from the EC's 7th Framework Programme (FP7/2007-2013), grant agreement 247947 (GARNICS).

\section{References}

[1] V. R. d. Angulo and C. Torras. Learning inverse kinematics via cross-point function decomposition. In Proceedings of the International Conference on Artificial Neural Networks, ICANN '02, pages 856-864, London, UK, UK, 2002. Springer-Verlag.

[2] A. Baranes and P.-Y. Oudeyer. Intrinsically motivated goal exploration for active motor learning in robots: A case study. In Intelligent Robots and Systems (IROS), 2010 IEEE/RSJ International Conference on, pages 1766 -1773, oct. 2010.
[3] D. A. Cohn, Z. Ghahramani, and M. I. Jordan. Active learning with statistical models. CoRR, cs.AI/9603104, 1996.

[4] J. S. de la Cruz, D. Kulić, and W. Owen. Online incremental learning of inverse dynamics incorporating prior knowledge. In Proceedings of the Second international conference on Autonomous and intelligent systems, AIS'11, pages 167176, Berlin, Heidelberg, 2011. Springer-Verlag.

[5] A. D'Souza, S. Vijayakumar, and S. Schaal. Learning inverse kinematics. In Intelligent Robots and Systems, 2001. Proceedings. 2001 IEEE/RSJ International Conference on, volume 1, pages 298 -303 vol.1, 2001.

[6] F. Larsson, E. Jonsson, and M. Felsberg. Simultaneously learning to recognize and control a low-cost robotic arm. Image Vision Computing, 27(11):1729-1739, Oct. 2009.

[7] D. Mitrovic, S. Klanke, and S. Vijayakumar. Adaptive optimal feedback control with learned internal dynamics models. In O. Sigaud and J. Peters, editors, From Motor Learning to Interaction Learning in Robots, volume 264 of Studies in Computational Intelligence, pages 65-84. Springer Berlin Heidelberg, 2010.

[8] J. J. Moré. The Levenberg-Marquardt algorithm: Implementation and theory. In G. A. Watson, editor, Numerical Analysis, pages 105-116. Springer, Berlin, 1977.

[9] E. Oyama, A. Agah, K. F. MacDorman, T. Maeda, and S. Tachi. A modular neural network architecture for inverse kinematics model learning. Neuro-computing, 38:797-805, 2001.

[10] E. Oyama, T. Maeda, J. Gan, E. Rosales, K. MacDorman, S. Tachi, and A. Agah. Inverse kinematics learning for robotic arms with fewer degrees of freedom by modular neural network systems. In Intelligent Robots and Systems, 2005. (IROS 2005). 2005 IEEE/RSJ International Conference on, pages $1791-1798$, aug. 2005.

[11] F. Pourboghrat and J.-C. Shiao. Neural networks for learning inverse kinematics of redundant manipulators. In Neural Networks, 1991., IJCNN-91-Seattle International Joint Conference on, volume ii, page 1004 vol.2, jul 1991.

[12] R. Saegusa, G. Metta, G. Sandini, and S. Sakka. Active motor babbling for sensorimotor learning. In Robotics and Biomimetics, 2008. ROBIO 2008. IEEE International Conference on, pages $794-799$, feb. 2009.

[13] S. Schaal and C. Atkeson. Robot juggling: implementation of memory-based learning. Control Systems, IEEE, 14(1):57 -71 , feb. 1994.

[14] S. Schaal and C. Atkeson. Learning control in robotics. Robotics Automation Magazine, IEEE, 17(2):20 -29, june 2010.

[15] B. Settles. Active learning literature survey. Technical report, 2009.

[16] B. Siciliano, L. Sciavicco, L. Villani, and G. Oriolo. Robotics - Modelling, Planning and Control. Springer, 2009.

[17] T. Sugihara. Solvability-unconcerned inverse kinematics based on levenberg-marquardt method with robust damping. In Humanoid Robots, 2009. Humanoids 2009. 9th IEEE-RAS International Conference on, pages 555 -560, dec. 2009.

[18] S. Vijayakumar, A. D'souza, and S. Schaal. Incremental online learning in high dimensions. Neural Comput., 17:26022634, December 2005. 